\title{
Sensitive Radio-Frequency Measurements of a Quantum Dot by Tuning to Perfect Impedance Matching
}

\author{
N. Ares, ${ }^{1}$ F. J. Schupp, ${ }^{1}$ A. Mavalankar, ${ }^{1}$ G. Rogers, ${ }^{1}$ J. Griffiths, ${ }^{2}$ G. A. C. Jones,${ }^{2}$ I. Farrer, ${ }^{2}$ \\ D. A. Ritchie, ${ }^{2}$ C. G. Smith, ${ }^{2}$ A. Cottet, ${ }^{3}$ G. A. D. Briggs, ${ }^{1}$ and E. A. Laird ${ }^{1}$ \\ ${ }^{1}$ Department of Materials, University of Oxford, Parks Road, Oxford OX1 3PH, United Kingdom \\ ${ }^{2}$ Cavendish Laboratory, J. J. Thomson Avenue, Cambridge CB3 OHE, United Kingdom \\ ${ }^{3}$ Laboratoire Pierre Aigrain, Ecole Normale Supérieure-PSL Research University, CNRS, Université \\ Pierre et Marie Curie-Sorbonne Universités, Université Paris Diderot-Sorbonne Paris Cité, \\ 24 rue Lhomond, 75231 Paris Cedex 05, France \\ (Received 30 November 2015; revised manuscript received 1 February 2016; published 24 March 2016)
}

\begin{abstract}
Electrical readout of spin qubits requires fast and sensitive measurements, which are hindered by poor impedance matching to the device. We demonstrate perfect impedance matching in a radio-frequency readout circuit, using voltage-tunable varactors to cancel out parasitic capacitances. An optimized capacitance sensitivity of $1.6 \mathrm{aF} / \sqrt{\mathrm{Hz}}$ is achieved at a maximum source-drain bias of $170-\mu \mathrm{V}$ rootmean-square and with a bandwidth of $18 \mathrm{MHz}$. Coulomb blockade in a quantum-dot is measured in both conductance and capacitance, and the two contributions are found to be proportional as expected from a quasistatic tunneling model. We benchmark our results against the requirements for single-shot qubit readout using quantum capacitance, a goal that has so far been elusive.
\end{abstract}

DOI: 10.1103/PhysRevApplied.5.034011

\section{INTRODUCTION}

Measuring the quantum state of an electronic device with high fidelity requires sensitive, fast, and noninvasive readout. If the state can be mapped to an electrical impedance, this goal can be achieved using radio-frequency reflectometry of an electrical resonator incorporating the quantum device [1]. This technique permits rapid readout of charge sensors [2,3], spin qubits [4], and nanomechanical resonators [5], as well as complex impedance measurements of quantum-dot circuits [6-11]. For optimal sensitivity, which can approach the quantum limit [12], impedance matching between the device and the external circuitry is essential to maximize power transfer between them $[13,14]$. This requirement is made challenging by the large resistance typical of quantum-dot devices $(\gtrsim 100 \mathrm{k} \Omega$, compared with usual line impedance $Z_{0}=50 \Omega$ ) and by parasitic capacitances in the matching circuit which vary unpredictably between devices.

We present a circuit that achieves controllable perfect matching with a high device impedance, even accounting for parasitics. Voltage-tunable capacitors allow in situ tuning of the matching condition $[15,16]$ and an absolute calibration of the capacitance sensitivity. We measure the complex impedance of a Coulomb-blockaded quantum dot, finding that the capacitance changes in proportion to the conductance. This relation is in agreement with a quasistatic model of electron tunneling.

\section{REFLECTOMETRY WITH PERFECT IMPEDANCE MATCHING}

We implement the matching scheme using as the device under test a gate-defined GaAs quantum dot measured in a dilution refrigerator as shown in Fig. 1(a). The impedance matching network is realized with a chip inductor $L$ and capacitors $C_{M}, C_{D}$, and $C_{S}$ forming a resonant circuit incorporating the device. To make a reflectometry measurement, a radio-frequency signal with frequency $f_{C}$ injected at port 1 of the cryostat is coupled via a directional coupler to the matching network input. The reflected amplified signal is returned to port 2. From the amplitude and phase of this signal, the reflection coefficient of the resonant circuit and, therefore, the complex impedance of the device can be deduced. A room-temperature homodyne detection circuit demodulates a chosen quadrature of the reflected signal to a de signal $V_{D}$. Simultaneous dc transport measurements are made using a tee to apply a bias voltage $V_{\text {bias }}$.

In previous work $[1-3,6,7,15]$, the impedance match is usually hindered by parasitic capacitances. Even with careful engineering, sample wiring typically contributes a sample capacitance $C_{S} \gtrsim 0.3 \mathrm{pF}$ in parallel with the device [19]. In our experiment, these parasitic capacitances are mitigated by adding a matching capacitor $C_{M}$ and a decoupling capacitor $C_{D}$ at the input of the matching network. This principle is illustrated in Figs. 1(b)-1(e), which show simulated reflection coefficient $\Gamma$ as a function of frequency for typical device parameters. With no matching capacitor [Figs. 1(b) and 1(c)], perfect matching (indicated by zero reflection) is achieved only for one value of $C_{S}$, in this case, $0.14 \mathrm{pF}$. With a parasitic capacitance above this value, perfect matching cannot be achieved at any carrier frequency $f_{C}$, degrading the sensitivity. One approach to restore matching is to increase the inductance 
(a)

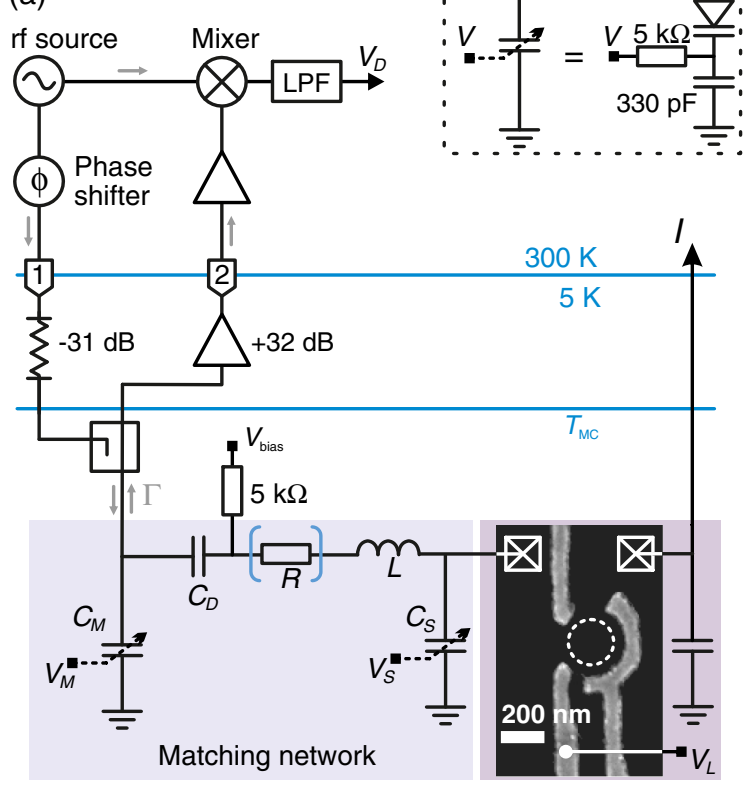

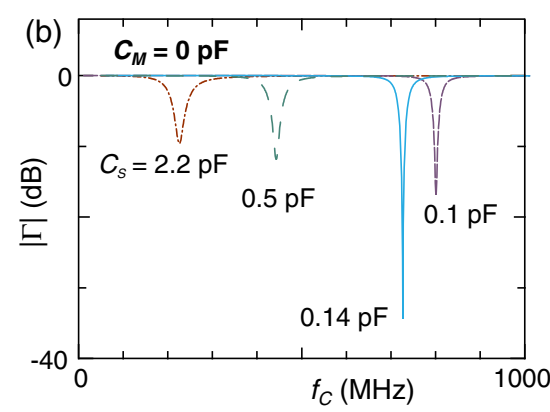
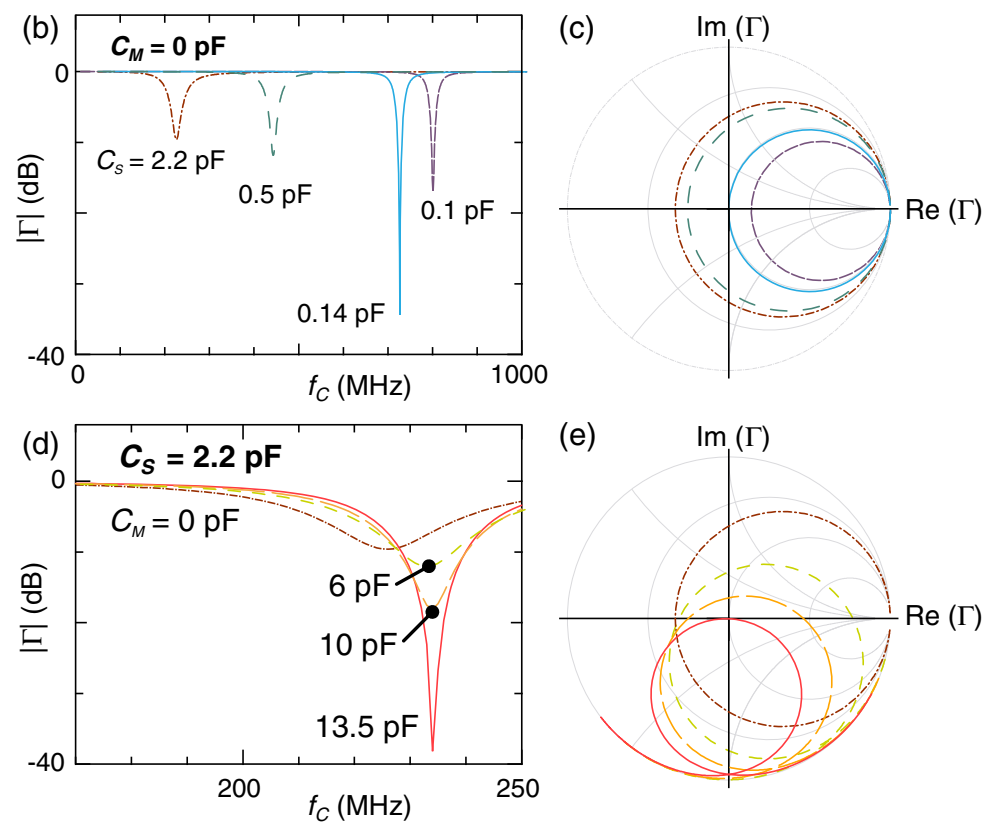

(e)

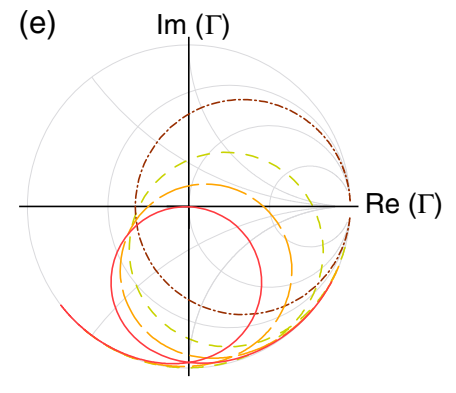

FIG. 1. (a) Experimental setup. A gate-defined quantum dot (electron micrograph right, with ohmic contacts denoted by boxes) is coupled to an impedance-matching network formed from an inductor $L\left(223 \mathrm{nH}\right.$ ), variable capacitors $C_{S}$ and $C_{M}$ (tuned through the circuit shown in the inset), and fixed capacitor $C_{D}(87 \mathrm{pF})$. Parasitic losses in the circuit are parametrized by an effective resistance $R$. To probe the matching network, a radio-frequency signal is injected at port 1, passed via a directional coupler, and after reflection and amplification received at port 2 . The reflected signal is demodulated at room temperature to a dc voltage $V_{D}$ by mixing it with a local oscillator; by adjusting the phase shift $\phi$, different quadratures of the signal can be detected. Alternatively, the signal is measured using a network analyzer or spectrum analyzer. A bias resistor allows measurements of the device current $I$ with dc bias $V_{\text {bias. }}$. (b), (c) Simulation with no matching capacitor $\left(C_{M}=0\right)$. Voltage reflection coefficient $\Gamma$ is plotted as a function of frequency for different values of sample capacitance $C_{S}$, as magnitude (b), and as a Smith chart [17] (c). The effective resistance is taken as $R=20 \Omega$, the device resistance as $1 \mathrm{G} \Omega$, and specified nonidealities of the inductor are included [18]. The capacitance of the device is taken as included in $C_{S}$. Perfect matching occurs when $\Gamma$ crosses the origin of the Smith chart $(|\Gamma|=0)$. With these parameters, perfect matching is achieved only when $C_{S}=0.14 \mathrm{pF}$, less than typical parasitic values. (d),(e) Simulated reflection for varying $C_{M}$. Perfect matching can be achieved even for a realistic large value of $C_{S}$ (here at $C_{M}=13.5 \mathrm{pF}$ for $C_{S}=2.2 \mathrm{pF}$ ). In (c) and (e), gray contours on the Smith chart indicate constant real or imaginary circuit input impedance.

$L$; however, this reduces the readout bandwidth, and more problematically introduces self-resonances of the inductor close to the operating frequency. Our approach is to introduce the capacitor $C_{M}$ to cancel out a reactive contribution to the impedance. By increasing $C_{M}$, a perfect match can be achieved even with a much larger value of $C_{S}$ [Figs. 1(d) and 1(e)]. In this scheme, the purpose of $C_{D}$ is to increase slightly the quality factor $Q$ of the circuit by decoupling it from the input.

This scheme is implemented using varactors (Macom MA46H204-1056) for $C_{M}$ and $C_{S}$ controlled by voltages $V_{M}$ and $V_{S}$, so that the parameters of the matching network can be tuned in situ (Fig. 2). The device under test is a laterally defined quantum dot [20] fabricated by patterning $\mathrm{Ti} / \mathrm{Au}$ gates over a GaAs/AlGaAs heterostructure containing a two-dimensional electron gas (depth $90 \mathrm{~nm}$, mobility $125 \mathrm{~m}^{2} \mathrm{~V}^{-1} \mathrm{~s}^{-1}$, carrier concentration $1.31 \times 10^{15} \mathrm{~m}^{-2}$ ). The device chip is bonded to a printed circuit board mounted with components of the matching circuit. Bias voltages for the varactors and the quantum-dot gates are applied through filtered wires with a bandwidth of approximately $100 \mathrm{kHz}$. A bias tee (not shown) allows a high-frequency signal to be added to $V_{L}$ for characterization at higher frequency.

At a refrigerator temperature $T_{\mathrm{MC}}=1 \mathrm{~K}$, gate voltages are set to pinch off the quantum dot completely (device resistance $>200 \mathrm{M} \Omega$ ). The quality of the impedance match in this configuration is probed by measuring the transmission $S_{21}$ between ports 1 and 2, which is proportional to $\Gamma$. With $C_{M}$ set to the upper end of its range $\left(C_{M} \sim 14 \mathrm{pF}\right)$, Fig. 2(a) shows $S_{21}$ as a function of frequency for different values of $V_{S}$. As $V_{S}$ is increased, the resonance frequency $f_{0}$ increases, confirming the change in $C_{S}$. The quality of the match depends strongly on $C_{S}$, with a minimum in the reflected power near $V_{S}=13.5 \mathrm{~V}$. From fits to these data using a simple circuit model, parameters can be estimated as follows [18]: From the trace with $V_{S}=13.5 \mathrm{~V}$, the tuned capacitances $C_{M}$ and $C_{S}$, the effective resistance $R$ characterizing parasitic losses, and the cable insertion loss can be extracted. Traces for other values of $V_{S}$ are then well reproduced using only $C_{S}$ and $R$ as free parameters. Perfect matching is achieved at $f_{C} \approx 211 \mathrm{MHz}$ and, according to 


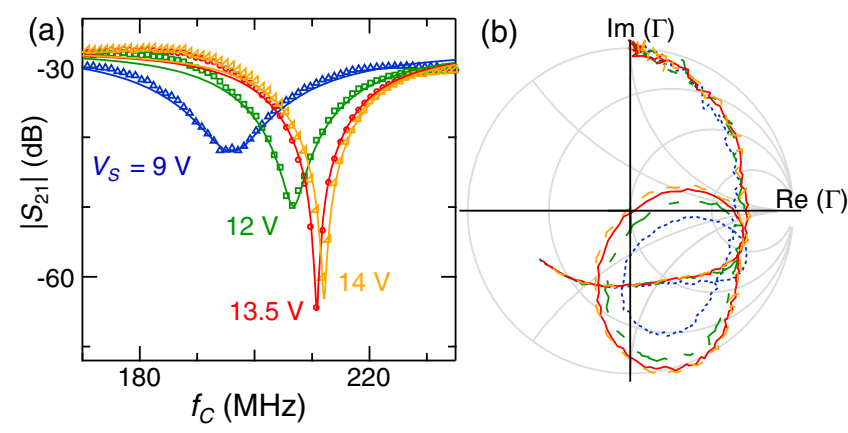

FIG. 2. Reflectometry measurements as a function of frequency for different $V_{S}$ settings. Data are taken at $T_{\mathrm{MC}}=1 \mathrm{~K}$ with $V_{M}$ held constant. (a) Magnitude of $S_{21}$, with data (symbols) fitted with a circuit model (lines). (b) The same data converted to circuit reflectance and plotted on a Smith chart. Perfect matching is achieved for $V_{S}=13.5 \mathrm{~V}$. The direction in which the traces cross the origin of the Smith chart is opposite to Fig. 1(c) because the effective resistance $R$ also changes with $V_{S}$.

this model, with $C_{S} \approx 2.78 \mathrm{pF}$. Using the inferred insertion loss and the known amplifier gain, which give the proportionality constant between $S_{21}$ and $\Gamma$, the complex reflection coefficient $\Gamma$ can be plotted on a Smith chart [Fig. 2(b)]. As $V_{S}$ is tuned, the traces cross the origin, confirming that the minimum seen in Fig. 2(a) indeed indicates a perfect match.

\section{CHARACTERIZING THE CAPACITANCE SENSITIVITY}

The ability to tune the circuit into perfect matching allows for highly sensitive capacitance measurements. This high sensitivity to capacitive changes is demonstrated in Fig. 3, which characterizes the sensitivity by detecting the response to a known capacitance change. A sinusoidal signal with root-mean-square (rms) amplitude $V_{m}$ and frequency $f_{m}=1.75 \mathrm{kHz}$ is added to $V_{S}$ to modulate $C_{S}$ by a known amount $\delta C$ [18]. Modulating $C_{S}$, we guarantee that the response is purely capacitive, unlike modulations on the quantum-dot impedance that result in both a capacitive and a resistive response.

As a result of this modulation of $C_{S}$, the power $P$ detected at port 2 shows sidebands at $f_{C} \pm f_{m}$ [Fig. 3(a)]. From the height of the sidebands above the noise floor, the sensitivity is given by $S_{C}=(1 / \sqrt{2}) \delta C(\Delta f)^{-1 / 2} 10^{-\mathrm{SNR} / 20}$, where SNR is the sideband signal-to-noise ratio expressed in decibels, and $\Delta f$ is the resolution bandwidth [21]. Over the range of varactor settings measured, $S_{C}$ is found to change by a factor $>15$, with the best sensitivity close to perfect matching as expected [Fig. 3(b)]. This dependence is reproduced well by the same circuit model as above [18]. In agreement with the model, $S_{C}$ is optimized when $f_{C}$ is set to the resonance frequency $f_{0}$ [Fig. 3(c)]. The optimum sensitivity attained at $V_{S}=13.5 \mathrm{~V}$ and $f_{C}=f_{0}=$ $210.75 \mathrm{MHz}$ is $S_{C}=1.6 \mathrm{aF} / \sqrt{\mathrm{Hz}}$.
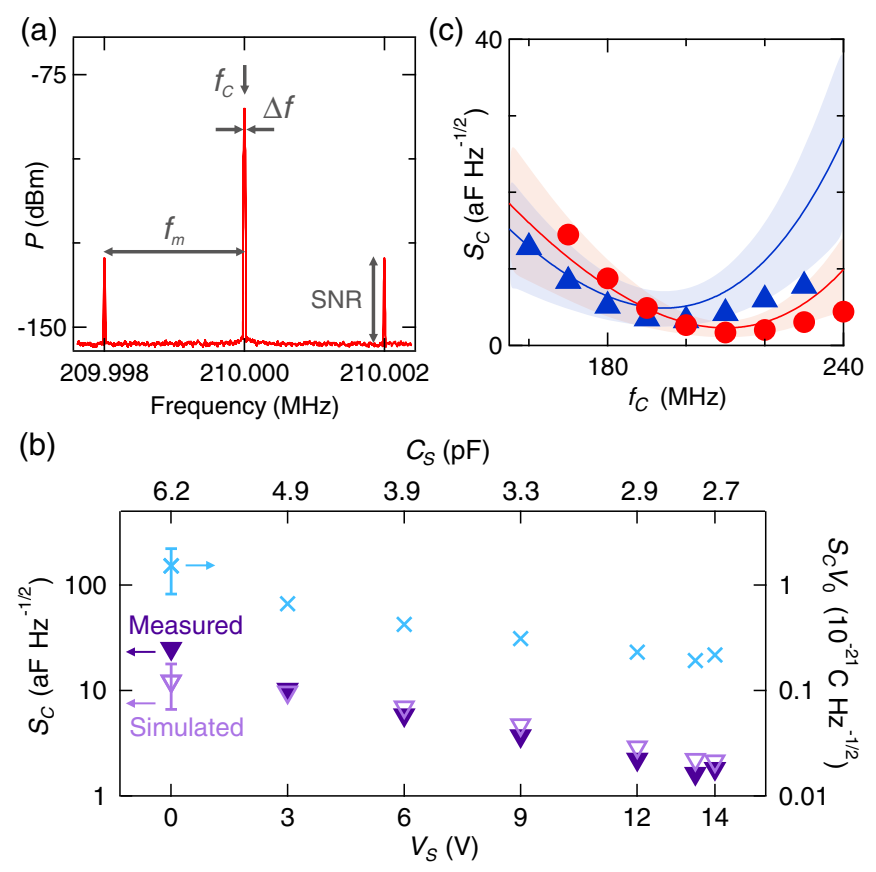

FIG. 3. (a) Power spectrum of signal at port 2 near perfect matching $\left(V_{S}=13.5 \mathrm{~V}\right)$ with varactor modulation $V_{m}=2 m V_{\text {rms }}$ showing the carrier peak and modulation sidebands. The SNR and resolution bandwidth $(\Delta f)$ are indicated. (b) Left axis: Capacitance sensitivity $S_{C}$ as a function of $V_{S}$ measured (filled triangles) and simulated (empty triangles). Agreement is good except for $V_{S} \leq 3 \mathrm{~V}$, where $f_{0}$ approaches a resonance of the cryostat. Right axis: Figure of merit $S_{C} V_{0}$ (crosses) as a function of $V_{S}$. Measurement parameters are $\Delta f=10 \mathrm{~Hz}, V_{m}=2 m V_{\text {rms }}$, $f_{m}=1.75 \mathrm{kHz}$, and $f_{C}=f_{0}$. Tuning the circuit near perfect matching improves the sensitivity to $S_{C}=1.6 \mathrm{aF} / \sqrt{\mathrm{Hz}}$. Fitted values of $C_{S}$ at each $V_{S}$ setting are marked on the top axis. Error bars on the data are smaller than the symbols. Error bars for $S_{C} V_{0}$ and simulated $S_{C}$ reflect systematic uncertainty in the power delivered to the matching network [18]. For clarity, only a single error bar is marked. (c) Symbols: Measured $S_{C}$ as a function of $f_{C}$ for $V_{S}=13.5 \mathrm{~V}$ (circles) and $V_{S}=9 \mathrm{~V}$ (triangles). Curves: Simulated $S_{C}$. Shaded bands indicate systematic uncertainty in the simulation of the same origin as in (b).

In characterizing the sensitivity, it is crucial to take account of measurement backaction. With larger applied power or improved matching, the capacitance sensitivity can be improved at the price of a larger voltage drop across the device, potentially disturbing the state being measured. The figure of merit is, therefore, not simply $S_{C}$ but the product $S_{C} V_{0}$, where $V_{0}$ is the rms excitation voltage at the device [18]. This figure of merit is plotted in Fig. 3(b), with $V_{0}$ calculated from the carrier power using the circuit parameters from Fig. 2. For all data in Fig. 3, the carrier power $P_{1}$ at port 1 is set to $-29 \mathrm{dBm}$ and near perfect matching $V_{0}=117 \pm 54 \mu V_{\text {rms }}$; i.e., the maximum bias applied is approximately $170 \mu V_{\text {rms }}$. The figure of merit $S_{C} V_{0}$ is minimized at the same circuit tuning as $S_{C}$, confirming that the optimal configuration of the circuit 
is, indeed, close to perfect matching. Note that minimizing $S_{C} V_{0}$ is not achieved by minimizing $C_{S}$ but by setting $C_{S}$ to achieve perfect matching.

\section{MEASURING THE QUANTUM-DOT IMPEDANCE}

We now turn to measurements of the quantum dot. First, we confirm that the impedance of the device itself can be measured with good sensitivity and bandwidth. Gate voltages are adjusted to the flank of one Coulomb peak at a point of maximum transconductance. With a modulation voltage now applied to a gate, Fig. 4(a) shows the sideband SNR as a function of $f_{C}$ for two different varactor settings. Again, the perfect matching condition (still corresponding to $V_{S}=13.5 \mathrm{~V}$ ) yields a bigger SNR. Figure 4(b) shows the SNR as a function of $f_{m}$, from which the readout bandwidth can be extracted; this readout bandwidth is found to be $34 \mathrm{MHz}$ at $V_{S}=9 \mathrm{~V}$ and $18 \mathrm{MHz}$ at $V_{S}=13.5 \mathrm{~V}$. These data confirm that the readout bandwidth is set by the $Q$ factor of the circuit and that the tradeoff between bandwidth and sensitivity can be tuned via a varactor.

Next, the stability diagram of the quantum dot is measured [18]. With the circuit cooled to $T_{\mathrm{MC}}=20 \mathrm{mK}$, simultaneous measurements of the dc transport conductance and the demodulated signal $V_{D}$ are shown as a function of $V_{L}$ and $V_{\text {bias }}$ [Figs. 4(c) and 4(d)]. Coulomb-blockade diamonds are evident in both Figs. 4(c) and 4(d). The similarity between these plots shows that changes in the quantum-dot impedance are well captured in reflectometry.

Below $1 \mathrm{~K}$, GaAs junction varactors show a delayed response to the tuning voltage, making acoustic-frequency modulation unreliable at $T_{\mathrm{MC}}=20 \mathrm{mK}$. Nevertheless, they continue to act as a radio-frequency capacitor, although with diminished and temperature-dependent tuning range. For this reason, exact impedance matching could not be achieved at $20 \mathrm{mK}$ on this cooldown, that will require increasing the geometrical contribution to $C_{S}$ or decreasing that to $C_{M}$.

Although the setup is optimized for capacitance sensitivity measurements, we can also operate our device as a single-electron transistor and estimate its charge sensitivity. The charge sensitivity is calculated from the measured SNR at a given bandwidth and the charge modulation estimated from the modulation amplitude and the Coulomb peak spacing [1], in an analogous expression to the one used for $S_{C}$. We obtain approximately $1650 \mu \mathrm{e} / \sqrt{\mathrm{Hz}}$ with a maximum $V_{0}$ of $144 \mu V_{\mathrm{rms}}$. For Si transistors, the state-of-theart value of $37 \mu e \mathrm{~Hz}^{-1 / 2}$ is achieved with an applied voltage to the rf gate of $0.5 \mathrm{mV}$ [11]. Our diminished charge sensitivity reflects the smaller rf bias, the smaller lever arm, and the lifetime broadening of the Coulomb peaks with respect to Ref. [11].

In the data of Fig. 4(d), the demodulated signal is sensitive to both conductance and capacitance of the
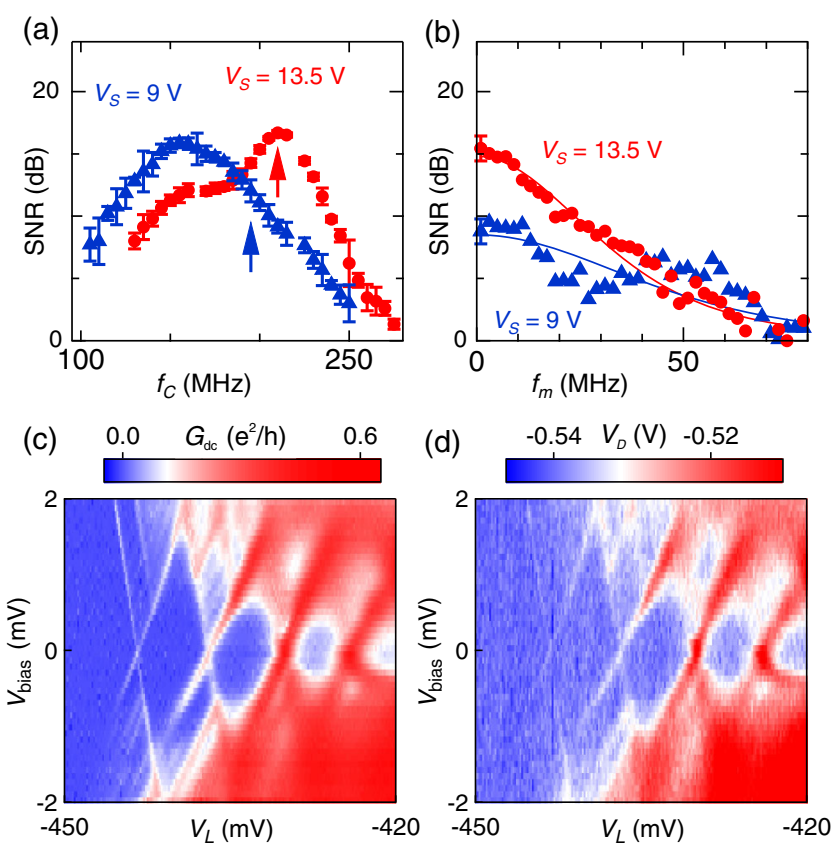

FIG. 4. (a),(b) Comparison of SNR close to perfect matching $\left(V_{S}=13.5 \mathrm{~V}\right)$ and away from perfect matching $\left(V_{S}=9 \mathrm{~V}\right)$, with modulation applied to the gate voltage $V_{L}$. SNR is plotted against carrier frequency in (a) with $f_{m}=1.1 \mathrm{MHz}$, and against modulation frequency in (b) with $f_{C}=f_{0}$ [marked with arrows in (a)] where $f_{0}$ is measured as in Fig. 2(a). For both data sets, $\Delta f=10 \mathrm{~Hz}$, the modulation amplitude is $0.48 \mathrm{mV}_{\mathrm{rms}}$ and $T_{\text {MC }}=1 \mathrm{~K}$. The applied power in (a) is adjusted at each frequency and voltage setting so as not to broaden the Coulomb peaks [18]; in (b), the power is fixed at the value chosen for $f_{C}=f_{0}\left(P_{1} \approx-31 \mathrm{dBm}\right.$ both at 9 and $\left.13.5 \mathrm{~V}\right)$. As seen from (a), the SNR is maximized near perfect matching and for $f_{C} \approx f_{0}$, although a resonance of the cryostat at low frequency enhances the SNR around $f_{C}=160 \mathrm{MHz}$. From Lorentzian fits [curves in (b)], the $3-\mathrm{dB}$ readout bandwidth can be extracted. Error bars indicate variation between different data sets in (a) and the uncertainty in the noise level in (b); for clarity, only a single error bar is marked in (b). (c) Conductance through the quantum dot as a function of $V_{\text {bias }}$ and $V_{L}$ measured at $T_{\mathrm{MC}}=20 \mathrm{mK}$ and showing Coulomb-blockade diamonds. (d) Demodulated voltage $V_{D}$ measured simultaneously with (c) with $V_{S}=13.5 \mathrm{~V}$ $\left(f_{C}=210.75 \mathrm{MHz}\right)$. The applied power $P_{1}=-40 \mathrm{dBm}$ does not broaden the Coulomb peaks at $T_{\mathrm{MC}}=20 \mathrm{mK}$.

quantum device. To isolate the capacitance $C_{\text {dot }}$, we measure $V_{D}$ as a function of the phase shift $\phi$ applied in the demodulation circuit. Figure 5(a) shows traces measured on and off a Coulomb peak, showing the phase shift associated with the quantum capacitance. To extract $C_{\text {dot }}$ from the measured phase shift, it is not sufficient simply to assume they are proportional because changes in the quantum-dot conductance also lead to a phase shift; however, using the measured dc conductance $G_{\mathrm{dot}}^{\mathrm{dc}}$ within our circuit model, it is possible to calculate $C_{\mathrm{dot}}$ [18]. Figure 5(b) shows $C_{\mathrm{dot}}$ calculated at each gate voltage over a series of Coulomb peaks, together with $G_{\mathrm{dot}}^{\mathrm{dc}}$ measured at 
(a)

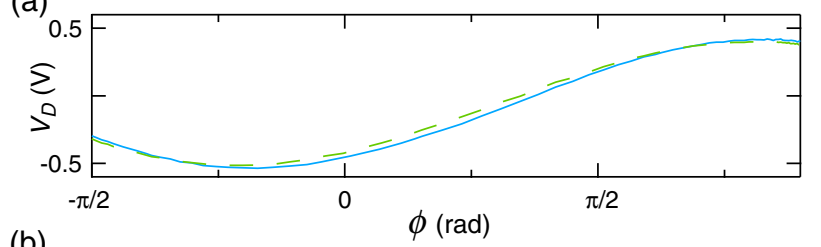

(b)

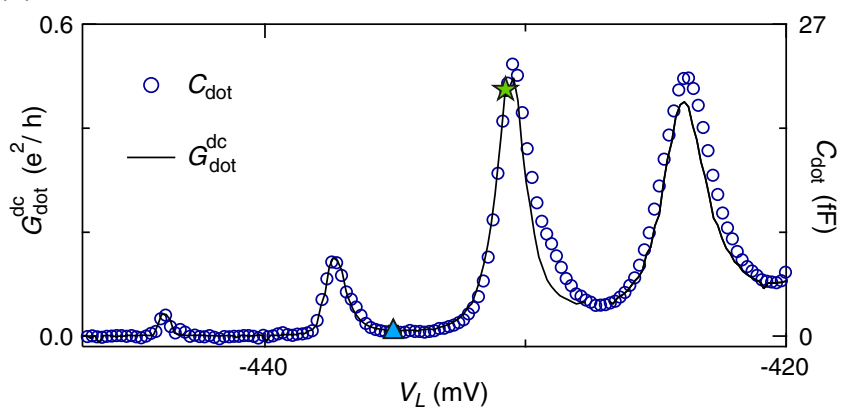

FIG. 5. (a) Demodulated response of the circuit as a function of $\phi$ for $V_{L}$ set on (green dashed) and off (blue solid) a Coulomb peak with $V_{\text {bias }}=0$. Both the amplitude and phase of $V_{D}$ are different, indicating a dissipative as well as a dispersive contribution on peak. (b) Imaginary contribution to the device impedance as a function of $V_{L}$ over a series of Coulomb peaks. Line: Measured dc conductance from Fig. 4(c). A gap in the line shows that we account for a switch event that occurs after the data in Fig. 4(c) are taken. Symbols: Capacitance extracted from curves as in (a) and a circuit model of the matching network. $C_{\text {dot }}$ varies proportionally to $G_{\mathrm{dot}}^{\mathrm{dc}}$ as a function of $V_{L}$.

the same settings. $C_{\mathrm{dot}}$ can, in principle, be calculated only from the amplitude and phase of the curves in Fig. 5(a). However, while $\phi$ corresponds directly to the phase of $\Gamma$, the amplitude requires a conversion factor that due to small nonlinearities in the demodulation circuit we cannot consistently determine. We, thus, rely on $\phi$ and $G_{\mathrm{dot}}^{\mathrm{dc}}$ to extract $C_{\mathrm{dot}}$.

In our data, it is evident that the quantum-dot capacitance is proportional to the conductance. This should not be true in the general case. However, such a behavior is possible in the asymmetric limit $\Gamma_{R} \ll \Gamma_{L}$ with $\Gamma_{R}$ approximately constant with $V_{L}$, where we expect both $C_{\mathrm{dot}}$ and $R_{\mathrm{dot}}$ to vary with $V_{L}$ like the density of states of the quantum dot $[18,22,23]$. Interestingly, in Ref. [24], a similar behavior is observed for a quantum dot coupled to a coplanar microwave cavity, i.e., the phase shift in the cavity microwave transmission, which reveals $C_{\mathrm{dot}}$ is approximately proportional to the dc conductance of the dot (see Ref. [24], Fig. 2).

This proportionality contrasts with previous measurements where the tunnel barriers are more opaque and nonproportionality between $\mathrm{dc}$ or ac conductance and capacitance can be observed $[7,25]$. This is the case, for instance, when the quantum-dot dynamics is dominated by the quantum charge-relaxation effect [26] evidenced in $\mathrm{rf}$ conductance measurements. This rich phenomenology [27-30] can be explored with our setup.

\section{DISCUSSION}

These sensitive measurements of quantum-dot impedance are promising for readout of singlet-triplet spin qubits in a double quantum dot. Using quantum capacitance for readout of a singlet-triplet obviates the need for a charge sensor [6], which is attractive for scalable two-dimensional architectures. However, although the theoretical sensitivity of this technique [31] allows for single-shot readout in a few microseconds, practical sensitivities are found to be well below this, in part because of poor impedance matching.

Estimating the difference in quantum capacitance [6] between qubit states as approximately $10 \mathrm{fF}$, our measured sensitivity $S_{C}=1.6 \mathrm{aF} / \sqrt{\mathrm{Hz}}$ would, at first sight, indicate single-shot readout with the unit SNR in integration time $T_{\text {meas }} \sim 13$ ns. Crucially, this sensitivity is achieved with a maximum bias $V_{0} \approx 170 \mu V_{\text {rms }}$, which is smaller than the typical singlet-triplet splitting in a qubit device [32] and, therefore, does not induce charge relaxation in the triplet manifold. However, this calculation does not take into account the fact that the quantum capacitance peaks in a narrow bias range near zero detuning. The single-shot readout time should, instead, be estimated by comparing the product $S_{C} V_{0}$, which characterizes the sensitivity to charge induced on the source electrode, with the actual charge $\lambda e$ induced by electron tunneling, where $\lambda$ is the lever arm. Taking $\lambda=0.3$ from Fig. 4 and the mean value of $S_{C} V_{0}$ close to perfect matching, we find that the unit SNR requires $T_{\text {meas }} \sim 64 \mu \mathrm{s}$. Since this is about twice the singlet-triplet qubit relaxation time [33] in GaAs, further improvements are required to achieve single-shot readout. Our approach can be improved by optimizing the remaining geometric capacitance in the circuit by using superconducting inductors to increase the quality factor [10,34] and by using a superconducting amplifier with drastically reduced noise temperature [35].

\section{ACKNOWLEDGMENTS}

We acknowledge discussions with $\mathrm{M}$. Shea and J. Medford. Research at Oxford University is supported by EPSRC (Grant No. EP/J015067/1), DSTL, the European Union, and the Royal Academy of Engineering. This publication is also made possible through support from Templeton World Charity Foundation. The opinions expressed in this publication are those of the authors and do not necessarily reflect the views of Templeton World Charity Foundation.

[1] R. J. Schoelkopf, The radio-frequency single-electron transistor (rf-set): A fast and ultrasensitive electrometer, Science 280, 1238 (1998).

[2] M. C. Cassidy, A. S. Dzurak, R. G. Clark, K. D. Petersson, I. Farrer, D. A. Ritchie, and C. G. Smith, Single shot charge 
detection using a radio-frequency quantum point contact, Appl. Phys. Lett. 91, 222104 (2007).

[3] D. J. Reilly, C. M. Marcus, M. P. Hanson, and A. C. Gossard, Fast single-charge sensing with a rf quantum point contact, Appl. Phys. Lett. 91, 162101 (2007).

[4] C. Barthel, M. Kjærgaard, J. Medford, M. Stopa, C. M. Marcus, M. P. Hanson, and A. C. Gossard, Fast sensing of double-dot charge arrangement and spin state with a radiofrequency sensor quantum dot, Phys. Rev. B 81, 161308 (2010).

[5] M. D. LaHaye, J. Suh, P. M. Echternach, K. C. Schwab, and M. L. Roukes, Nanomechanical measurements of a superconducting qubit, Nature (London) 459, 960 (2009).

[6] K. D. Petersson, C. G. Smith, D. Anderson, P. Atkinson, G. A. C. Jones, and D. A. Ritchie, Charge and spin state readout of a double quantum dot coupled to a resonator, Nano Lett. 10, 2789 (2010).

[7] S. J. Chorley, J. Wabnig, Z. V. Penfold-Fitch, K. D. Petersson, J. Frake, C. G. Smith, and M. R. Buitelaar, Measuring the Complex Admittance of a Carbon Nanotube Double Quantum Dot, Phys. Rev. Lett. 108, 036802 (2012).

[8] M. Jung, M. D. Schroer, K. D. Petersson, and J. R. Petta, Radio frequency charge sensing in InAs nanowire double quantum dots, Appl. Phys. Lett. 100, 253508 (2012).

[9] M. D. Schroer, M. Jung, K. D. Petersson, and J. R. Petta, Radio Frequency Charge Parity Meter, Phys. Rev. Lett. 109, 166804 (2012).

[10] J. I. Colless, A. C. Mahoney, J. M. Hornibrook, A. C. Doherty, H. Lu, A. C. Gossard, and D. J. Reilly, Dispersive Readout of a Few-Electron Double Quantum Dot with Fast rf Gate Sensors, Phys. Rev. Lett. 110, 046805 (2013).

[11] M. F. Gonzalez-Zalba, S. Barraud, A. J. Ferguson, and A. C. Betz, Probing the limits of gate-based charge sensing, Nat. Commun. 6, 6084 (2015).

[12] W. W. Xue, Z. Ji, F. Pan, J. Stettenheim, M. P. Blencowe, and A. J. Rimberg, Measurement of quantum noise in a single-electron transistor near the quantum limit, Nat. Phys. 5, 660 (2009).

[13] A. Aassime, G. Johansson, G. Wendin, R. J. Schoelkopf, and P. Delsing, Radio-Frequency Single-Electron Transistor as Readout Device for Qubits: Charge Sensitivity and Backaction, Phys. Rev. Lett. 86, 3376 (2001).

[14] T. Hasler, M. Jung, V. Ranjan, G. Puebla-Hellmann, A. Wallraff, and C. Schönenberger, Shot Noise of a Quantum Dot Measured with Gigahertz Impedance Matching, Phys. Rev. Applied 4, 054002 (2015).

[15] T. Müller, B. Küng, S. Hellmüller, P. Studerus, K. Ensslin, T. Ihn, M. Reinwald, and W. Wegscheider, An in situ tunable radio-frequency quantum point contact, Appl. Phys. Lett. 97, 202104 (2010).

[16] S. Hellmüller, M. Pikulski, T. Müller, B. Küng, G. PueblaHellmann, A. Wallraff, M. Beck, K. Ensslin, and T. Ihn, Optimization of sample-chip design for stub-matched radiofrequency reflectometry measurements, Appl. Phys. Lett. 101, 042112 (2012).

[17] D. M. Pozar, Microwave Engineering (John Wiley and Sons, New York, 2011).

[18] See the Supplemental Material at http://link.aps.org/ supplemental/10.1103/PhysRevApplied.5.034011 for details of the circuit simulation, how the circuit sensitivity is measured and simulated, how the carrier signal power is chosen, and the quantum-dot capacitance estimation and discussion about its proportionality to the quantum-dot conductance.

[19] J. M. Hornibrook, J. I. Colless, A. C. Mahoney, X. G. Croot, S. Blanvillain, H. Lu, A.C. Gossard, and D. J. Reilly, Frequency multiplexing for readout of spin qubits, Appl. Phys. Lett. 104, 103108 (2014).

[20] A. Mavalankar, S. J. Chorley, J. Griffiths, G. A. C. Jones, I. Farrer, D. A. Ritchie, and C. G. Smith, A non-invasive electron thermometer based on charge sensing of a quantum dot, Appl. Phys. Lett. 103, 133116 (2013).

[21] H. Brenning, S. Kafanov, T. Duty, S. Kubatkin, and P. Delsing, An ultrasensitive radio-frequency single-electron transistor working up to $4.2 \mathrm{~K}$, J. Appl. Phys. 100, 114321 (2006).

[22] Y. Meir, N. S. Wingreen, and P. A. Lee, Transport through a Strongly Interacting Electron System: Theory of Periodic Conductance Oscillations, Phys. Rev. Lett. 66, 3048 (1991).

[23] Ya. M. Blanter and M. Büttiker, Shot noise in mesoscopic conductors, Phys. Rep. 336, 1 (2000).

[24] M. R. Delbecq, V. Schmitt, F. D. Parmentier, N. Roch, J. J. Viennot, G. Fève, B. Huard, C. Mora, A. Cottet, and T. Kontos, Coupling a Quantum Dot, Fermionic Leads, and a Microwave Cavity on a Chip, Phys. Rev. Lett. 107, 256804 (2011).

[25] J. Gabelli, G. Fève, J.-M. Berroir, B. Plaçais, A. Cavanna, B. Etienne, Y. Jin, and D. C. Glattli, Violation of Kirchhoff's laws for a coherent RC circuit, Science 313, 499 (2006).

[26] M. Büttiker, H. Thomas, and A. Prétre, Mesoscopic capacitors, Phys. Lett. A 180, 364 (1993).

[27] S. E. Nigg, R. López, and M. Büttiker, Mesoscopic Charge Relaxation, Phys. Rev. Lett. 97, 206804 (2006).

[28] Y. Hamamoto, T. Jonckheere, T. Kato, and T. Martin, Dynamic response of a mesoscopic capacitor in the presence of strong electron interactions, Phys. Rev. B 81, 153305 (2010).

[29] M. Filippone, K. Le Hur, and C. Mora, Giant Charge Relaxation Resistance in the Anderson Model, Phys. Rev. Lett. 107, 176601 (2011).

[30] A. Cottet, T. Kontos, and B. Douçot, Electron-photon coupling in mesoscopic quantum electrodynamics, Phys. Rev. B 91, 205417 (2015).

[31] G. Johansson, L. Tornberg, and C. M. Wilson, Fast quantum limited readout of a superconducting qubit using a slow oscillator, Phys. Rev. B 74, 100504 (2006).

[32] J. R. Petta, A. C. Johnson, J. M. Taylor, E. A. Laird, A. Yacoby, M. D. Lukin, C. M. Marcus, M. P. Hanson, and A.C. Gossard, Coherent manipulation of coupled electron spins in semiconductor quantum dots, Science 309, 2180 (2005).

[33] C. Barthel, D. J. Reilly, C. M. Marcus, M. P. Hanson, and A. C. Gossard, Rapid Single-Shot Measurement of a Singlet-Triplet Qubit, Phys. Rev. Lett. 103, 160503 (2009).

[34] H. O. H. Churchill, Ph.D. thesis, Harvard University, 2012.

[35] J. Stehlik, Y.-Y. Liu, C. M. Quintana, C. Eichler, T. R. Hartke, and J. R. Petta, Fast Charge Sensing of a CavityCoupled Double Quantum Dot Using a Josephson Parametric Amplifier, Phys. Rev. Applied 4, 014018 (2015). 